\title{
Nanoparticules méfalliques
}

\author{
Marc LAMY de la CHAPELLE \\ Laboratoire CSPBAT UMR7244 - UFR Santé, Médecine et Biologie Humaine - Université Paris 13 \\ marc.lamydelachapelle@ univ-paris 13.fr
}

Au cours des dix dernières années, de nombreuses recherches ont porté sur le développement de nouveaux moyens de détection de composés chimiques ou biologiques à la fois rapides, sensibles et à faible coût, l'objectif étant alors d'atteindre des seuils de détection inférieurs aux méthodes classiques (dosage immuno-enzymatique par exemple dont les seuils de détection sont de l'ordre du $\mathrm{nM} / \mathrm{L}$ ). Pour améliorer les performances des capteurs, une des solutions envisagées est d'exploiter les propriétés optiques des nanoparticules métalliques. Ainsi, sous certaines conditions, ces nanostructures peuvent provoquer une exaltation très importante du champ proche électromagnétique. Cette exaltation du champ va alors induire une exaltation de toute information optique (diffusion, émission) provenant d'un analyte situé à proximité de ces nanoparticules.

\section{Propriétés optiques de nanoparticules métalliques}

La sensibilité d'un capteur optique à base de nanoparticules (NP) métalliques sera directement liée aux propriétés optiques des NP et elle sera d'autant plus élevée que l'exaltation du champ sera importante. Il est donc primordial de pouvoir maîtriser cette exaltation à travers le contrôle de quelques paramètres clés liés à la géométrie et à la nature de la NP.

\section{Spécificité des matériaux métalliques}

La spécificité des matériaux métalliques est que leurs électrons de conduction sont faiblement liés au noyau des atomes. Ces électrons peuvent donc se déplacer facilement autour de leur position d'équilibre et être aisément excités sous l'effet d'une onde électromagnétique. La réponse optique d'un matériau sous l'effet d'un champ électromagnétique incident est décrite par sa permittivité relative $\varepsilon_{\mathrm{r}}$.

De plus, dans le cas particulier des NP, leur taille devient comparable à la profondeur de peau de pénétration d'une onde électromagnétique dans un métal (à $633 \mathrm{~nm}$, cette profondeur de peau est de $126 \mathrm{~nm}$ pour l'or et de $202 \mathrm{~nm}$ pour l'argent). En conséquence, l'ensemble des électrons conducteurs de la NP va interagir avec le champ électromagnétique incident. L'énergie absorbée par la NP va alors induire une oscillation collective des électrons due à l'interaction de Coulomb à l'échelle des NP. Ce phénomène est appelé plasmon de surface localisé (PSL).

Une onde électromagnétique interagissant avec une NP peut être absorbée ou diffusée. Dans le cas d'une NP sphérique, les rendements de diffusion, d'absorption ainsi que d'extinction (diffusion + absorption) peuvent être prédits par la théorie classique de Mie, qui fournit la solution exacte des équations de Maxwell.

\section{Interaction rayonnement-nanoparticule}

Cette théorie permet ainsi de calculer la polarisabilité $\alpha$ de la NP. Dans le cas particulier de l'approximation quasistatique ou dipolaire, la taille de la NP est inférieure à I/10 de $\lambda$, la longueur d'onde d'excitation de la lumière. L'amplitude du champ est alors supposée comme constante dans toute la NP, et pour une sphère noyée dans un milieu environnant ayant une permittivité $\varepsilon_{1}$, a s'écrit :

$$
\alpha=4 \pi \varepsilon_{0} \varepsilon_{r} R^{2}\left(\frac{\varepsilon_{r}-\varepsilon_{1}}{\varepsilon_{r}+2 \varepsilon_{1}}\right)
$$

Il est alors possible de s'apercevoir que cette polarisabilité sera maximale lorsque $\operatorname{Re}\left(\varepsilon_{\mathrm{r}}\right)=-2 \varepsilon_{1}$. Cette condition est alors appelée condition de résonance et est notamment atteinte lorsque l'énergie d'oscillation du PSL correspond à l'énergie du champ incident.

La position de la résonance de PSL (RPSL) peut se mesurer expérimentalement par spectroscopie d'extinction. Le maximum du spectre d'extinction est alors caractéristique de la RPSL et correspond à une longueur d'onde particulière (figure 1).

Dans le cas de la sphère dans l'air $\left(\varepsilon_{1}=1\right)$, cette condition sera remplie pour une longueur d'onde de $354 \mathrm{~nm}$ pour une NP d'argent et de $484 \mathrm{~nm}$ pour une NP d'or.

\section{Influence de la permittivité du milieu ambiant}

D'après l'équation 1, la permittivité du milieu environnant $\varepsilon_{1}$ (et l'indice de réfraction correspondant $\mathrm{n}_{1}$ ) joue un rôle essentiel dans la détermination des conditions de résonance car elle détermine pour quelle valeur de $\operatorname{Re}\left(\varepsilon_{\mathrm{r}}\right)$ la condition de résonance est satisfaite. En conséquence, une augmentation de l'indice de réfraction entourant la NP conduit à une diminution de $\operatorname{Re}\left(\varepsilon_{\mathrm{r}}\right)$. La condition de résonance est alors modifiée et conduit à un décalage vers le rouge de la RPSL. Cela est notamment dû à l'accumulation de charges dans le diélectrique entourant la NP, ce qui induit un amortissement du PSL. 


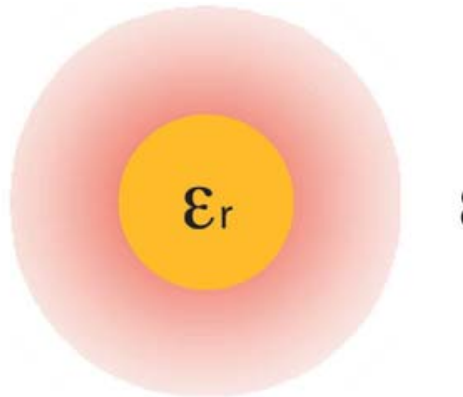

$\varepsilon_{1}$
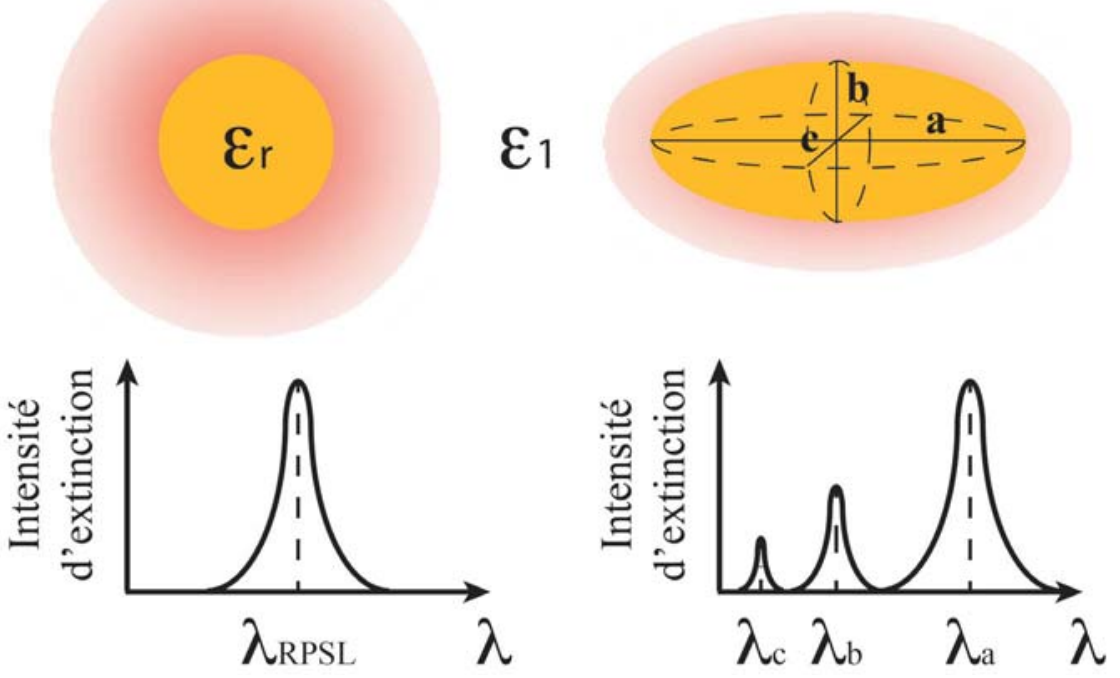

Figure 1. Représentation schématique de l'exaltation du champ autour de nanoparticules sphérique (gauche) ou ellipsoïdale (droite) avec les RPSL associées (spectres d'extinction en dessous). La constante diélectrique du métal est $\varepsilon_{\mathrm{r}}$ et la constante diélectrique du milieu extérieur est $\varepsilon_{1}$.

\section{Influence de la forme des nanoparticules}

Dans le cas des formes sphéroïdes ayant trois demi-axes différents $(j=x, y$, et $z)$, chaque axe exhibera sa propre polarisabilité $\alpha J$ tel que:

$\alpha=12 \pi \varepsilon_{0} \varepsilon_{r} a b c\left(\frac{\varepsilon_{r}+\varepsilon_{1}}{3 L_{j} \varepsilon_{r}+\varepsilon_{1}\left(3-3 L_{j}\right)}\right)$

avec $L_{j}$ le facteur de dépolarisation selon l'axe $J$ de la NP ( $L_{J}=1 / 3$ pour une sphère).

Ayant trois polarisabilités différentes selon les trois axes, une NP sphéroïdale présente trois RPSL, une pour chaque axe, et le spectre d'extinction présente alors trois maxima distincts correspondant à chacune des RPSL. La RPSL dont la longueur d'onde est la plus faible correspond à l'axe le plus court de la NP alors que la RPSL de longueur d'onde la plus grande correspond à l'axe le plus grand (figure 1).

De plus, lorsque la dimension d'un axe diminue par rapport aux autres (augmentation de $L_{j}$ ), on observe alors un décalage vers le bleu de sa RPSL. À l'inverse, l'augmentation de la dimension d'un des axes (diminution de $L_{j}$ ) induit le décalage vers le rouge de la RPSL (figure 2).

La forme est donc un paramètre important pour contrôler les propriétés optiques des NP. Il est alors possible d'accorder la RPSL à la longueur d'onde souhaitée simplement en modifiant la forme de la NP.

\section{Influence de la taille des nanoparticules}

La taille est aussi un paramètre important mais pour de grandes NP (typiquement supérieure à $40 \mathrm{~nm}$ pour l'or), l'approximation quasi-statique n'est plus valable et doit être corrigée car la taille des NP n'est plus négligeable par rapport à la longueur d'onde incidente. En conséquence :

- les électrons conducteurs n'oscillent plus exactement en phase le long de la NP, ce qui conduit à une diminution du champ de dépolarisation (cet effet est connu sous le nom d'effet de retard

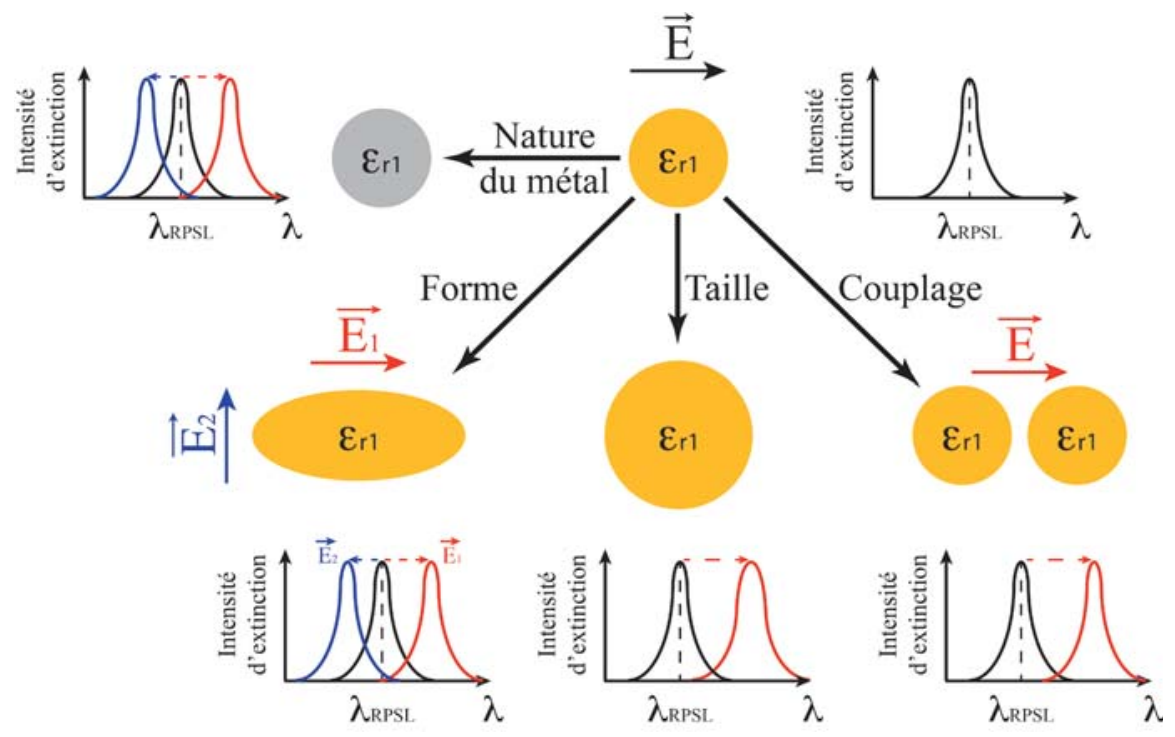

Figure 2. Principaux paramètres influençant la position de la RPSL. et est proportionnel au carré de la taille de la NP),

- les pertes par rayonnement (rayonnement d'amortissement) ne sont plus négligeables et induisent un amortissement de la RPSL (cet effet est proportionnelà la puissance trois de la taille de la NP).

Ces deux phénomènes vont avoir une influence sur la RPSL et vont induire, respectivement, un décalage vers le rouge et un élargissement de la RPSL lorsque la taille de la NP augmente. Par conséquent, la taille de la NP est un troisième paramètre clé qui gouverne la condition de résonance (figure 2 ).

\section{Influence de la distance entre les nanoparticules}

Un dernier paramètre géométrique va influencer la position de la RPSL : c'est la distance entre NP. Si l'écart entre les particules est très faible par rapport à la longueur d'onde incidente, on observe alors un couplage champ proche des PSL des deux NP. Une diminution de l'écart entre les NP conduit à un décalage de la RPSL vers le rouge pour un champ incident polarisé le long de l'axe de couplage, tandis qu'un décalage vers le bleu est observé pour une polarisation perpendiculaire (figure 2).

Ce dernier cas présente un intérêt important car le couplage induit un confinement du champ sur quelques nanomètres entre les NP et crée des "points chauds». 


\section{Influence du choix du métal}

Le choix du métal est également un point important car les changements de constantes diélectriques d'un métal à l'autre vont induire des modifications de la condition de résonance pour une même taille et une même forme de NP. De nombreux métaux tels que $\mathrm{Pd}, \mathrm{Pt}, \mathrm{Al}, \mathrm{Au}$, Ag présentent des RPSL. Toutefois, l'or et l'argent sont les métaux les plus choisis car ils présentent des RPSL très intenses dans la gamme spectrale du visible (figure 2).

En résumé, les principaux paramètres qui régissent les propriétés optiques des NP sont le milieu environnant, la taille, la forme, la distance inter-particules et le matériau. Le contrôle de l'ensemble de ces paramètres permet un contrôle précis de la position de la RPSL.

\section{Exaltation du champ local}

Lorsque la NP est excitée à une longueur d'onde proche de la condition de résonance, il se crée alors un champ électromagnétique local à proximité de la surface. Sous certaines conditions, I'amplitude de ce champ est exaltée par rapport à l'intensité du champ incident, cette exaltation pouvant être 100 à 1000 fois supérieure à l'amplitude du champ incident. Il se crée alors une nanosource de lumière extrêmement intense en champ proche et il est possible de l'exploiter comme une excitation locale à une échelle de l'ordre de la taille de la NP. Ainsi, une des caractéristiques de ce champ local est son confinement extrême sur des distances de l'ordre de quelques dizaines de nanomètres. En effet, l'amplitude de ce champ décroit très rapidement, de façon exponentielle, à partir de la surface de la NP. Les longueurs typiques de décroissance de ce champ sont de l'ordre de 10 à $20 \mathrm{~nm}$ (voire moins) pour des nanostructures dont les tailles sont de l'ordre de quelques dizaines de nanomètres de diamètre ou de longueur. Le maximum d'exaltation est alors atteint à la surface des NP. De plus cette exaltation dépend de plusieurs paramètres optiques et géométriques.

\section{Influence des paramètres optiques}

Tout d'abord, elle est fonction de la longueur d'onde d'excitation et de sa valeur par rapport à la longueur d'onde de RPSL. Intuitivement, il est logique de penser que pour maximiser l'interaction entre la lumière excitatrice et la NP, il est nécessaire que la longueur d'onde d'excitation soit proche de la RPSL. En fait, cette règle n'est pas valable sur l'ensemble de la gamme du visible. En effet, l'exaltation locale étant un phénomène champ proche et la mesure par spectroscopie d'extinction de la RPSL se faisant en champ lointain, il est possible d'observer un décalage entre la RSPL et la longueur d'onde pour laquelle le maximum d'exaltation champ proche est obtenu. Ce décalage (vers le rouge de l'excitation par rapport à la RPSL) est d'autant plus marqué que la RPSL se situe dans le proche infrarouge.

\section{Influence de la forme des nanostructures}

Une autre manière de maximiser l'exaltation champ proche est de créer des nanostructures ayant des formes allongées ou bien des structures locales en formes de pointes (triangles, étoiles...). En effet, dans ce cas précis, ces pointes vont favoriser l'accumulation locale de charges à leur extrémité et y créer un champ très intense. Ce phénomène est connu sous le nom d'effet paratonnerre et permet d'accroître l'exaltation en un point précis de la nanostructure.

\section{Couplage de plusieurs nanostructures}

Dernièrement, une autre méthode pour atteindre de fortes exaltations est de coupler entre elles deux ou plusieurs nanostructures. Comme nous l'avons vu précédemment, lorsque la distance entre deux NP est inférieure à quelques dizaines de nanomètres, il y a possibilité de recouvrement des champs proches des deux NP et donc couplage électromagnétique. Dans ce cas, lorsque le champ électrique incident est polarisé selon l'axe de couplage entre les deux NP, il se crée un champ très intense à l'intérieur de l'espace situé entre les NP. Il se forme alors des " points chauds » (ou hot-spots en anglais).

Ces phénomènes d'exaltation vont pouvoir être utilisés pour exalter tout phénomène optique se produisant à proximité de la ou des NP. Nous nous attarderons plus particulièrement à l'exploitation de ces exaltations locales dans le cadre du développement de nanocapteurs.

\section{Principe des capteurs exaltés}

Le principe d'un capteur est de détecter ou d'identifier un composé chimique ou biologique (analyte) et de mesurer le nombre d'analytes présents dans le milieu environnant. À l'heure actuelle, un des challenges en termes de développement de capteurs est d'améliorer la sensibilité de ces systèmes pour être en mesure de détecter des concentrations d'analytes de plus en plus faibles. Les seuils de sensibilité visés sont de l'ordre du pM/L voire des concentrations inférieures. Les phénomènes d'exaltation peuvent alors être exploités pour augmenter la sensibilité de capteurs optiques.

Cette exaltation du champ peut être utilisée de deux manières différentes pour la détection ou l'identification d'un analyte (figure 3).

\section{Comparaison avec les capteurs SPR (surface plasmon resonance)}

En premier lieu, d'après l'équation (1), la position de la RPSL va être extrêmement sensible à toute modification de la constante diélectrique externe à la NP et donc à toute modification de son environnement immédiat. Une augmentation de la constante diélectrique (>1) provoquée par un changement de l'environnement local (par exemple un solvant ou une molécule) va induire un décalage de la RPSL vers le rouge. La mesure d'un tel décalage va alors indiquer la présence d'une molécule à proximité de la surface de la NP. Ces capteurs sont en fait basés sur le même principe que les systèmes dits SPR (surface plasmon resonance) mais apportent des améliorations significatives comparativement aux performances des systèmes SPR. Premièrement, la taille des particules est à l'échelle nanométrique ce qui permet une localisation de la détection à une échelle équivalente. Il est ainsi possible d'envisager qu'une NP soit vue comme un nanocapteur individuel. L'utilisation de réseaux de NP permettrait alors la détection d'un très grand nombre d'analytes simultanément sur une surface 


\section{Capteur RPSL}

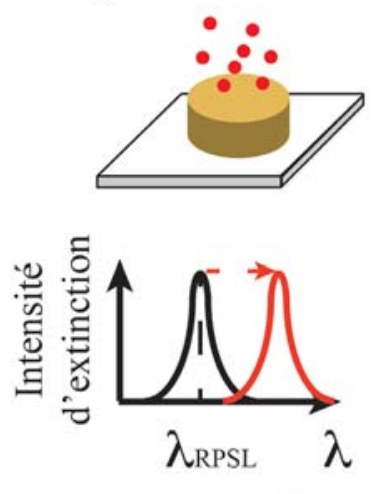

Capteur MEF

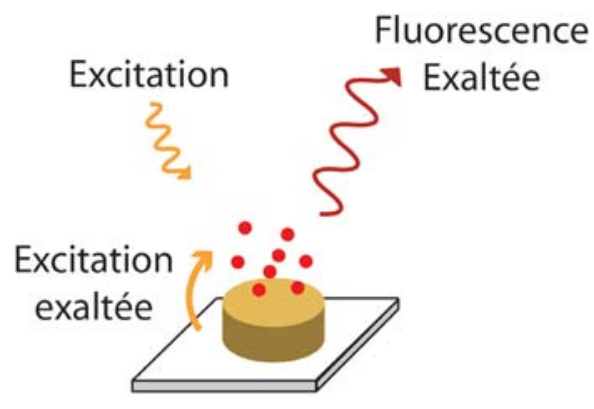

Capteur SERS

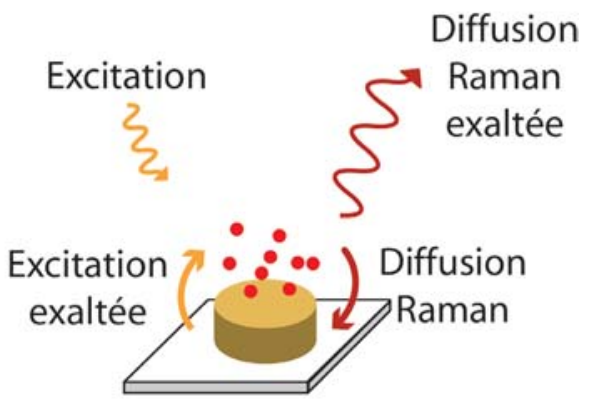

- Analyte

\section{Nanoparticule}

Figure 3. Schémas de principe des capteurs basés sur la RPSL, le MEF ou le SERS. extrêmement faible, démultipliant les possibilités de multiplexage. Deuxièmement, la longueur de décroissance du champ exalté et de l'amplitude du PSL est de l'ordre de 10 à $20 \mathrm{~nm}$. Comparativement, la longueur de décroissance en SPR est de l'ordre de $300 \mathrm{~nm}$ soit plus de 10 fois plus que pour les PSL. La RPSL est donc beaucoup plus sensible à toute modification de l'environnement local et permet la détection de petites molécules dont le poids moléculaire est inférieur à $1000 \mathrm{~g} / \mathrm{Mol}$ qui est la limite actuelle des systèmes SPR.

\section{Utilisation des processus d'interaction lumière-matière}

Deuxièmement, d'autres capteurs optiques sont basés sur un processus d'interaction lumière-matière telles que la fluorescence ou la diffusion Raman. Dans ces deux cas, le signal optique collecté dépend de l'intensité du rayonnement excitateur. Ces processus sont alors exaltés par la présence d'un champ électromagnétique exalté créé par les PSL. Ces processus exaltés sont connus sous les dénominations suivantes : fluorescence exaltée par un métal (metal enhanced fluorescence, MEF) et diffusion Raman exaltée de surface (surface enhanced raman scattering, SERS).

\section{Fluorescence exalfée}

Fondamentalement, la fluorescence consiste en l'absorption d'un photon dans les domaines spectraux du visible ou des ultraviolets, ce qui induit l'émission d'un deuxième photon de plus faible énergie. Ce phénomène est utilisé depuis de très nombreuses années pour la détection d'analytes en exploitant soit la fluorescence intrinsèque de la molécule à détecter, soit la fluorescence d'un marqueur (fluorophore) qui est lié à la molécule à détecter. Ce processus physique a longtemps été préféré au processus de diffusion Raman car la fluorescence a une section efficace beaucoup plus forte (12 à 14 ordres de grandeur) que celle de la diffusion Raman. Le signal de fluorescence obtenu est donc beaucoup plus intense que celui mesurable en diffusion Raman. La fluorescence peut bénéficier de l'exaltation du champ proche car ce dernier augmente l'absorption de la lumière par la molécule fluorescente et donc le rendement quantique (rapport entre le nombre de photons émis et absorbés) de fluorescence. Des exaltations de l'ordre de 10 à 100 ont été démontrées. Toutefois, la présence du métal à proximité de la molécule fluorescente peut entraîner un effet contraire nommé extinction (quenching en anglais). Par exemple, l'émission d'un fluorophore situé à une distance inférieure à $5 \mathrm{~nm}$ de la surface métallique est presque complètement éteinte. Ce phénomène est dû à l'absorption par la NP métallique de la fluorescence émise. Il est donc nécessaire d'optimiser la distance entre la molécule fluorescente à détecter et la surface métallique pour contrebalancer l'effet d'extinction par celui de l'exaltation.

\section{Diffusion Raman exaltée}

Depuis l'observation de molécules uniques en SERS à la fin des années 90, l'intérêt pour le développement de capteurs basés sur le SERS a été relancé. Le principal avantage dans le cas de tels capteurs est lié au fait que la diffusion Raman est une technique de détection sans marquage. En effet, la spectroscopie Raman est basée sur l'observation des vibrations moléculaires qui sont directement liées à la structure et à la composition chimique de la molécule observée. Dans ce cas, le spectre Raman peut être considéré comme une signature spectrale du composé étudié et ce spectre permet alors son identification. Le SERS est dû à un effet électromagnétique (facteur d'exaltation pouvant atteindre des valeurs allant jusqu'à $10^{8}$ ) et à un effet chimique (facteur d'exaltation de l'ordre de $10^{2}$ ). Même si ce dernier effet n'est pas encore complètement expliqué, il est lié à un transfert de charge entre la surface métallique et la molécule et/ou à la formation de liaisons chimiques entre le métal et la molécule. Les deux processus induisent alors une modification de la polarisabilité de la molécule et peuvent donc induire une augmentation de la diffusion Raman. Étant donné que l'exaltation associée à cet effet chimique est faible comparativement à celle atteinte par l'effet électromagnétique, cet effet est souvent négligé. La contribution électromagnétique est due à l'exaltation locale du champ proche au voisinage de la surface des NP. En fait, il a été démontré que cet effet est dûà l'interaction électromagnétique entre la molécule et la NP impliquant deux processus électromagnétiques distincts: - le premier provient de l'interaction du faisceau incident avec la NP. Il induit alors une exaltation locale du champ incident à la longueur d'onde excitatrice $\lambda_{0}$;

- le deuxième processus est expliqué par l'interaction de la diffusion Raman de la molécule avec la NP à la longueur 
d'onde $\lambda_{R^{\prime}}$ qui est décalée par rapport à $\lambda_{0}$. Ce second processus est appelé processus de re-radiation.

Le facteur d'exaltation total en SERS, $G\left(\lambda_{0}, \lambda_{R}\right)$, inclut donc deux facteurs d'exaltation aux deux longueurs d'onde $\lambda_{0}$ et $\lambda_{\mathrm{R}}$ tel que :

$I_{S E R S}=G\left(\lambda_{0}, \lambda_{R}\right) I_{R}=M^{2}\left(\lambda_{0}\right) M^{2}\left(\lambda_{R}\right) I_{R}$

où $I_{R}$ est l'intensité Raman mesurée en l'absence de NP métallique. Cette équation montre que le facteur d'exaltation totale dépend fortement de la position de la RPSL par rapport à la longueur d'onde d'excitation et au spectre Raman.

\section{Optimnisation de l'exalfation Ramam}

Il existe alors plusieurs règles à respecter en vue d'optimiser l'exaltation obtenue en SERS.

- Dans le cas de nanocylindres ou de nanotriangles, I'intensité SERS maximale est obtenue pour une position de la RPSL située entre $\lambda_{0}$ et $\lambda_{R}$.

- Lorsque $\lambda_{0}$ est située dans le proche infrarouge, la RPSL doit être décalée vers le bleu par rapport à $\lambda 0$ en raison du décalage champ proche/champ lointain.

Dans le cas de formes allongées, l'effet paratonnerre favorise le processus de re-radiation conduisant à un maximum d'intensité SERS pour une RPSL proche de $\lambda_{R}$.
- La direction de polarisation du champ incident influence fortement l'intensité SERS dans le cas de NP allongées.

- La longueur de décroissance du champ proche joue un rôle clé en SERS. II a ainsi été montré que l'éloignement de quelques nm des molécules à détecter entraine une diminution d'un ou de plusieurs ordres de grandeur de l'intensité SERS.

- Le couplage en champ proche entre les NP peut considérablement augmenter l'intensité SERS jusqu'à deux ordres de grandeur.

Au vu de l'ensemble de ces règles, il est donc indispensable de contrôler précisément les paramètres à la fois géométriques et optiques des NP utilisées pour optimiser l'exaltation en SERS et donc les performances d'un capteur basé sur le SERS. Les techniques de production des NP doivent être hautement reproductibles. C'est pour cela que les méthodes de nano-lithographie sont les plus souvent utilisées car elles permettent un excellent contrôle de la taille, de la forme ou de la distribution surfacique des NP. Une large gamme de techniques de nanolithographie peut être employée en utilisant des approches top-down (lithographies optique ou électronique) ou bottom-up (nano-impression ou lithographie par nanosphères).

\section{Performances \\ des capteurs exaltés}

La sensibilité fournie par l'exaltation du champ proche doit permettre la détection de petites quantités de substance à analyser. Ainsi, il est possible de trouver de nombreux exemples de détection d'espèces chimiques ou biologiques dans la littérature à partir de capteurs LSPR, MEF ou SERS. Pour les capteurs RPSL, les principaux résultats ont été obtenus sur des protéines (streptavidin, BSA, Concanavalin A...) avec des seuils de détection situés entre le $\mathrm{nM} / \mathrm{L}$ et le $\mathrm{pM} / \mathrm{L}$. Pour les capteurs MEF, une amélioration des performances des capteurs a été obtenue avec une diminution des seuils de détection d'un facteur 10 à 100. Pour finir, dans le cas des capteurs SERS, la détection de protéines (BSA, RNase $A$, myoglobine) a été testée avec des seuils de l'ordre du $\mu \mathrm{M} / \mathrm{L}$. Dans le cas de composés chimiques (R6G, BPE, glucose, benzenethiol...) des seuils de l'ordre du $\mathrm{nM} / \mathrm{L}$ ont été mesurés. Ces seuils sont globalement supérieurs à ceux atteints par la méthode RPSL mais les dernières méthodes d'optimisation des nanostructures permettent d'envisager des seuils de détection bien inférieurs pouvant descendre jusqu'à l'attomole par litre.

\section{- SPECTROGON}

State of the art products

\section{Filtres Interférentiels}

De 200 á $15000 \mathrm{~nm}$

- Passe-bande

- Passe-haut

- Passe-bas

- Large bande

- Densité neutre

- Disponible en stock

\section{Réseaux Holographiques}

De 150 á $2000 \mathrm{~nm}$

- Compression d'impulsion

- Télècom

- Accordabilité spectrale

- Monochromateurs

- Spectroscopie

- Disponible en stock 